\title{
Double-J Versus External Ureteral Stents in Kidney Transplantation: A Retrospective Analysis
}

\author{
Thomas Vogel ${ }^{1}$; Markus Utech ${ }^{2}$; Fabian Schmidt ${ }^{1}$; Wiebke Holscher Keplin ${ }^{1}$; Ricarda Diller ${ }^{3}$; \\ Jens Brockmann ${ }^{4}$; Heiner Wolters ${ }^{1,}$ \\ ${ }^{1}$ Department of General and Visceral Surgery, University Hospital Munster, Munster, Germany \\ ${ }^{2}$ Department of General and Visceral Surgery, Knappschafts Hospital, Recklinghausen, Germany \\ ${ }^{3}$ Department of General Surgery, Bruderkrankenhaus St. Josef, Paderborn, Germany \\ ${ }_{4}^{4}$ Organ Transplant Centre, King Faisal Specialist Hospital and Research Centre, Riyadh, Saudi Arabia \\ ${ }^{*}$ Corresponding author: Heiner Wolters, Department of General and Visceral Surgery, University Hospital Munster, Münster, Germany. Tel: +49-2518356301, Fax: +49-2518356402, \\ E-mail: heiner.wolters@ukmuenster.de
}

Received: February 8, 2015; Accepted: March 12, 2015

Background: Kidney transplantation has long been recognized as the best available therapy for end stage kidney disease. Objectives: This study aimed to compare outcomes of double-J versus percutaneous ureteral stent placement in renal transplantation. Patientsand Methods:Aretrospectiveanalysis wasperformed on data of renal transplantations performed atourinstitution ina12-month period. In this period, external and double-J stents were used in parallel. Length of hospital stay and stent-associated complications were evaluated.

Results: In 76 kidney transplants, 43 external (group 1) and 33 double-J (group 2) urinary stents were used. No significant difference was observed in the number of urinary tract infections, ureteric stenosis or necrosis. The mean overall length of hospital stay was comparable in both groups (20.7 days in group 1 vs 19.3 days in group $2, \mathrm{P}=0.533$ ). For patients without immunological complications, the hospital stay was significantly reduced using double-J stents (12.9 days in group $1,10.8$ days in group $2, \mathrm{P}=0.018$ ). Leakage of the ureteroneocystostomy occurred in 6 out of 43 patients in group 1(13.9\%). No case of anastomotic insufficiency was observed in group 2 ( $\mathrm{P}=0.035)$. Macrohematuria was detected in 13 out of the 43 patients in group 1 (30.2\%), compared to 3 out of 33 patients in group $2(9.1 \% ; \mathrm{P}=0.045)$.

Conclusions: This nonrandomized comparison of stent types in kidney transplantation supports the use of prophylactic double-J stents in terms of decreased ureteric complications and reduced length of hospital stay.

Keywords: Kidney Transplantation; Ureter; Stent

\section{Background}

Kidney transplantation has long been recognized as the best available therapy for end-stage kidney disease. While operative technique has been consistently refined over time and is now highly standardized, there is ongoing research on details of the procedure, possibly leading to a further reduction of postoperative complications and improvement of the long-term outcome.

Urologic complications after renal transplantation include urinary obstruction of the transplant ureter due to a variety of causes including stenosis or kinking of the transplant ureter, stenosis or insufficiency of the ureterovesical anastomosis, obstruction by blood clots from macrohematuria, and urinary tract infections (UTIs) (1).

Routine intraoperative ureteral stenting has been shown to reduce postoperative complications from stenosis or kinking of the transplant ureter and necrosis and insufficiency of the ureterovesical anastomosis (2-5). Routine urinary stenting has also been shown to be cost effective, at least if the stent is removed on day 30 after transplantation $(4,6)$.
However, there is an ongoing debate as to whether routine placement of a urinary stent could lead to an increase in a different set of postoperative complications including UTIs, vesicoureteral reflux, prolonged duration of postoperative macrohematuria, pressure necrosis of the ureter, obstruction of the stent leading to hydronephrosis of the donor organ, dislocation of the stent, and a troublesome foreign body sensation reported by some patients $(7,8)$.

Internal, double-J ureteric stents do not have an open leg outside of the body and could be expected to provide protection of urinary flow and could be expected to ensure urinary flow while providing protection to the ureterovesicular anastomosis, with a reduced rate of infectious complications when compared to externalized uretero-vesico-cutaneous stents. Additionally, due to their internal placement, double-J catheters can be left in situ for longer periods of time, possibly providing prolonged protection from postoperative complications (9). Percutaneous stents had been consistently used at our

Copyright (C) 2015, Nephrology and Urology Research Center. This is an open-access article distributed under the terms of the Creative Commons Attribution-NonCommercial 4.0 International License (http://creativecommons.org/licenses/by-nc/4.0/) which permits copy and redistribute the material just in noncommercial usages, provided the original work is properly cited. 
institution for the given advantages, including the ease of estimating the grafts excretory function, easy radiological examination of bladder and ureteroneocystostomy with contrast agent administered via the external opening, and simplicity of stent removal.

In light of mounting evidence for significantly reduced postoperative complications associated with the use of indwelling urinary stents in adult (9) and pediatric (10) renal transplant patients, our strategy concerning the use of ureteral stents changed to using double-J-style catheters for standard urinary stenting in kidney transplantation.

\section{Objectives}

This study aimed to compare the rate of postoperative complications occurring with both types of urinary stents to determine whether the use of internal JJ-type stents improves the outcome of adult renal transplantation when compared with external (uretero-vesico-cutaneous) stents.

\section{Patients and Methods}

This study was conducted as a retrospective analysis of all consecutive renal transplantations performed at our department within one year. From a total of 79 patients underwent renal transplantation during the study period, three patients who received multivisceral transplantations were excluded.

Details about the remaining 76 transplantations were retrieved from the renal transplant database, as well as by manual chart review of clinical, biochemical and radiological records. There were 61 primary transplants, 11 second transplants, 3 third transplants and one 4 th transplant in the series. Thirteen donor organs were from living-related donors and 63 from deceased organ donors.

A team of three experienced surgeons performed all transplantations using a single standardized operating technique. Donor organs were transplanted into the iliac fossa of the recipient using an extra peritoneal approach with arterial and venous anastomoses to the iliac vessels. Urinary continuity was established using a modified LichGregoire antireflux ureteroneocystostomy. All patients received intraoperative stenting of the transplant ureter, either by external percutaneous transcystic stenting (group 1, $\mathrm{n}=43$ ), or by internal (Double-J) stenting (group $2, \mathrm{n}=33$ ). Additionally, an indwelling transurethral bladder catheter was placed in all patients for an average of 6 days postoperatively.

Externally draining uretero-vesico-cutaneous stents were left in situ for a median of 10 days. Patients in group 2 had a $4.7 \mathrm{~F}, 8.22 \mathrm{~cm}$ double-J in-dwelling uretero-vesical stent (Cook Urological, Spencer, IN, USA) inserted for a median of 51 days.

Baseline immunosuppression consisted of calcineurin inhibitors (CsA or tacrolimus), corticosteroids and Mycophenolate Mofetil (MMF) and was given to all recipients. Additionally, induction therapy with anti-CD25 monoclo- nal antibodies (basiliximab $20 \mathrm{mg}$ ) was given in 22 cases in group 1 and 21 cases in group 2 on day 0 and day 4 .

Baseline serum creatinine levels were recorded preoperatively, and on a daily basis after transplantation. Color-coded duplex sonography was performed daily for the first postoperative week and as needed afterwards for the assessment of organ perfusion and vascular resistance, as well as for the exclusion of postoperative complications including hydroureter and/or hydronephrosis, hematoma and anastomotic insufficiency.

Endpoints of this study were postoperative complications including UTIs, ureteric stenosis or obstruction, anastomotic leakage and macrohematuria. In addition, the length of hospital stay was evaluated and compared between the two groups.

Diagnosis of urinary tract infection was based on was based on criteria specified by the centers for disease control and prevention (CDC) (11) and defined as a microbial count of more than $10^{5}$ microorganisms/ $\mu \mathrm{L}$ in conjunction with at least one of the following symptoms as experienced by the patient with no other recognized cause: fever $\left(>38^{\circ} \mathrm{C}\right)$, urgency, frequency, dysuria, or suprapubic tenderness. Ureteric stenosis was defined as a rise in serum creatinine by more than $20 \%$, ultrasonographic evidence for hydronephrosis and verification of the stenotic anastomosis by retrograde pyelography. If no relevant stenosis was discovered or there was direct evidence of obstruction by another cause (urolithiasis, thrombotic material), the complication was defined as ureteric obstruction. Anastomotic leakage was defined as any amount of contrast agent outside the transplant ureter or bladder detected by retrograde pyelography, routinely performed on postoperative day 8. Secondary macrohematuria was defined as any macrohematuria newly arising after cessation of initial postoperative hematuria.

Statistical analysis was performed using the SPSS statistical software package, (SPSS Inc., Chicago IL, USA). Group means or medians were compared using the unpaired t-test, contingency tables were analyzed using Fisher's exact test.

\section{Results}

In 76 kidney transplants 43 external (group1) and 33 double-J (group 2) stents were used. No significant demographic differences were observed between the two groups regarding patient age (median patient age of 50, range 18 - 73 for group 1 and a median age of 50, range 26 - 69 for group $2, \mathrm{P}=0.282)$, gender distribution $(30.2 \%$ females in group $1,42.4 \%$ in group $2, \mathrm{P}=0.998$ ), BMI (median 25.1 vs. 24.9 in group 1 and 2, respectively, $\mathrm{P}=0.795)$, percentage of living-related donors (20.9\% and $12.1 \%$ in groups 1 and 2, respectively.) and number of donors over the age of 65 taking part in the eurotransplant seniors program (ESP, $9.3 \%$ in group 1, and $12.1 \%$ in group 2 ). The duration of cold and warm ischemia in the donor organ did not differ significantly between the groups (Table 1).

Postoperative complication rates are shown in Table 2. 
Vogel Tet al.

There was no significant difference in the number of UTIs ( $\mathrm{n}=16$ or $37.2 \%$ in group $1, \mathrm{n}=17$ or $51.5 \%$ in group $2, \mathrm{P}=$ 0.481 ), ureteric stenosis ( $\mathrm{n}=3$ in group $1, \mathrm{n}=0$ in group 2 , $\mathrm{P}=0.256)$ or ureteric necrosis $(\mathrm{n}=1,2.3 \%$ in group $1, \mathrm{n}=0$ in group $2, \mathrm{P}=1.00$ ).

The overall mean length of hospital stay (Figure 1) was comparable in the two groups (19.3 days in group 1, 20.7 days in group $2, \mathrm{P}=0.533$ ). However, the hospital stay of pa- tients without immunological complications was significantly decreased using double-J stents (12.9 days in group 1 , 10.8 days in group $2, \mathrm{P}=0.018)$. Ureteroneocystostomyleakage occurred in 6 out of the 43 patients in group 2 (13.9\%), while no case of insufficient anastomosis was observed in group $1(\mathrm{P}=0.035)$. There were significantly fewer occurrences of secondary macrohematuria in group 1 (3/33 patients) as compared to group 2 (13 of 43 patients, $\mathrm{P}=0.045$ ).

\begin{tabular}{|c|c|c|c|}
\hline Characteristics & Group 1, External stent $(n=43)$ & Group 2, Double-J $(n=33)$ & PValue \\
\hline Median age at transplantation, (range) & $50(18-73)$ & $50(26-69)$ & 0.282 \\
\hline Gender & & & 0.998 \\
\hline Male & 30 & 19 & \\
\hline Female & 13 & 14 & \\
\hline Median BMI, kg/m² & $25.1 \pm 3.8$ & $24.9 \pm 4.1$ & 0.795 \\
\hline Donor origin -living: & & & 0.370 \\
\hline Related & 9 & 4 & \\
\hline Cadaveric & 34 & 29 & \\
\hline Old for old & 4 & 4 & 0.701 \\
\hline Cold ischemia, min & $658 \pm 335$ & $650 \pm 313$ & 0.919 \\
\hline Warm ischemia, min & $32 \pm 7$ & $30 \pm 9$ & 0.433 \\
\hline
\end{tabular}

a Abbreviations: BMI, body mass index, ESP, European Senior Program.

Table 2. Postoperative Complications

\begin{tabular}{lccc}
\hline Complication & Group 1, external stent $(\mathbf{n}=\mathbf{4 3})$ & Group 2, double-J $(\mathbf{n}=\mathbf{3 3})$ & PValue \\
\hline Urinary tract infection & $16(37.2)$ & $17(51.5 \%)$ & 0.481 \\
Ureteric stenosis & $3(6.98)$ & 0 & 0.256 \\
Ureteral necrosis & $1(2.33)$ & 0 & 1.000 \\
Urethral obstruction & $2(4.6)$ & 0 & 0.504 \\
Anastomotic leakage & $6(14)$ & 0 & 0.035 \\
Hematuria & $13(30.23)$ & $17(51.5)$ & 0.045 \\
\hline
\end{tabular}

A Length of hospital stay (all patients)

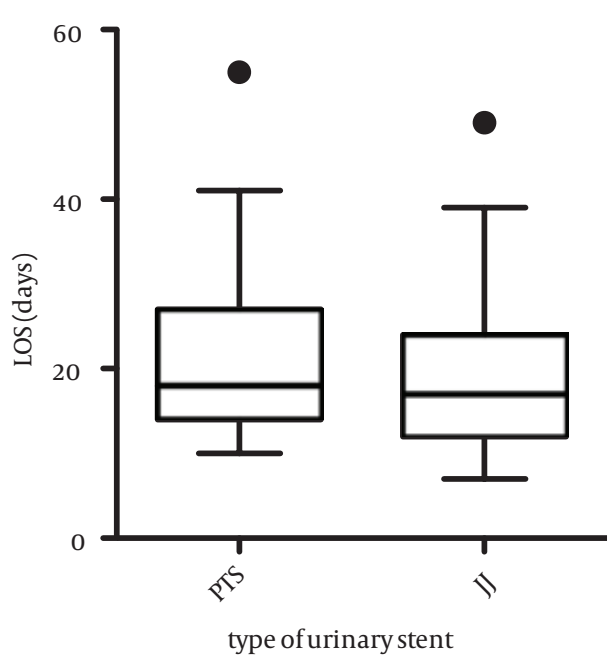

B

Length of stay, no immunological problems

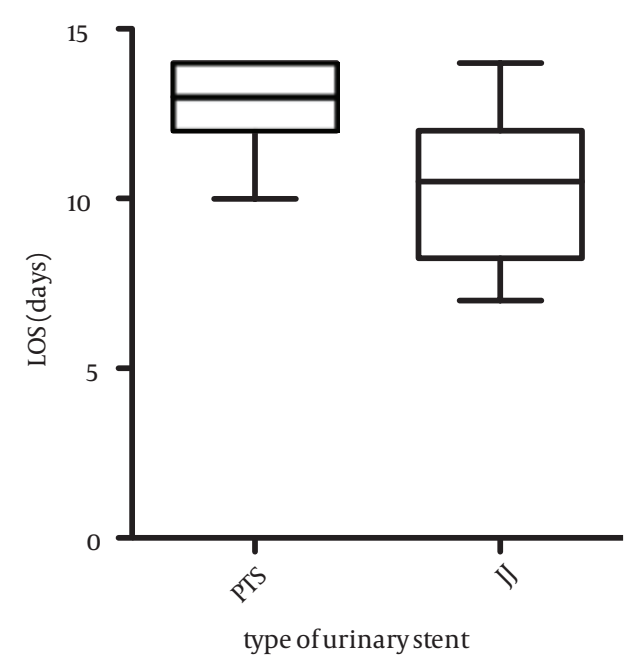

Figure 1. Length of Postoperative Hospital Stay in Days for Patients Without Immunological Complications (A) and With Immunological Complications Prolonging Length of Hospital Stay (LOS) (B) 


\section{Discussion}

The present study seems indicative of a lowered rate of postoperative urinary complications with the use of internally placed, double-J style ureterovesical stents when compared to percutaneous, uretero-vesico-cutaneous drainage.

Typical postoperative complications after kidney transplantation include obstruction, stenosis or kinking of the transplant ureter, insufficiency of the uretero-neovesicular anastomosis, as well as UTIs and postoperative macrohematuria, possibly leading to obstruction of urinary drainage and complications due to the heightened pressure on the transplant kidney as well as the anastomosis $(8,9,12-14)$.

In the present study, we have not seen a significant difference in the rate of ureteric stenosis, ureteric obstruction or anastomotic insufficiency between the two groups. However, it should be noted that none of these complications occurred in internally stented patients at all. Complication rates of $7 \%$ for ureteric stenosis and $4.6 \%$ for ureteric obstruction in the externally drained group are in line with prior studies (15), while an absence of each of these complications in the JJ group has previously been shown in large meta-analyses $(3,5)$.

Statistical relevance was shown concerning the rate of postoperative anastomotic insufficiency (14\% of externally drained patients vs. $0 \%$ of internally drained patients). These results are in line with prior studies on adult and juvenile transplant recipients. In these, the rate of postoperative anastomotic insufficiency was shown to be $\sim 10 \%$ in externally drained patients $(10,16-19)$, and nonexistent in patients with internal JJ-style drainage $(3,5)$. In our study, occurrence of all complications took place after removal of the external drainage; our hypothesis is that the longer duration of urinary stenting in the internally drained patients has a protective effect on the anastomosis. A recent study utilizing a 5-day external stenting protocol found this stenting period to be adequate for living donor transplant recipients only, while being insufficient for deceased donor transplantation (20).

We have found a high rate of secondary macrohematuria in patients with internal stents in the present study, while the rate of early postoperative hematuria did not differ between groups. No case of hematuria necessitated an operative revision, or led to a prolongation of hospital stay. In light of the protective effects of longer stent placement, hematuria may be an accepted disadvantage for the benefit of fewer complications.

The rate of postoperative UTIs did not differ significantly between patients with internal stenting and patients receiving external urinary drainage. In theory, externalized urinary stents could be accused of representing an additional entry point for microorganisms; therefore, heightening the risk of urinary tract infection, the additional placement of transurethral urinary catheters in recipients of JJ-style internal stents seems to suffice as an entryway, leading to the development of clinically significant UTIs (8).

In patients without immunological complications, length of hospital stay was significantly reduced in patients with JJ-stents as compared to externally drained patients, possibly reflecting the lower rate of urological complications in this group. In addition, JJ-style urinary stents do not have to be removed during the initial hospital stay, and later removal by cystoscopy can be performed in an outpatient setting.

In conclusion, the present study is indicative for an improved outcome with a lowered rate of postoperative urinary complications and shorter hospital stay of patients with internally placed, double-J uretero-vesical stents in kidney transplantation.

\section{Authors' Contributions}

Study concept and design: Ricarda Diller, Jens Brockmann, and Heiner Wolters; Acquisition of data: Thomas Vogel, Markus Utech, Fabian Schmidt, and Heiner Wolters; Analysis and interpretation of data: Thomas Vogel, Markus Utech, Fabian Schmidt, and Wiebke Holscher Keplin; Drafting of the manuscript: Thomas Vogel, Markus Utech, and Fabian Schmidt; Critical revision of the manuscript for important intellectual content: Heiner Wolters; Statistical analysis: Thomas Vogel, Markus Utech, and Wiebke Holscher Keplin; Administrative, technical, and material support: Heiner Wolters, Ricarda Dillerand Jens Brockmann; Study supervision: Jens Brockmann, and Heiner Wolters.

\section{References}

1. Buresley S, Samhan M, Moniri S, Codaj J, Al-Mousawi M. Postrenal transplantation urologic complications. Transplant Proc. 2008;40(7):2345-6.

2. Georgiev P, Boni C, Dahm F, Maurus CF, Wildi S, Rousson V, et al. Routine stenting reduces urologic complications as compared with stenting "on demand" in adult kidney transplantation. Urology. 2007;70(5):893-7.

3. Mangus RS, Haag BW. Stented versus nonstented extravesical ureteroneocystostomy in renal transplantation: a metaanalysis. Am J Transplant. 2004;4(11):1889-96.

4. Tavakoli A, Surange RS, Pearson RC, Parrott NR, Augustine T, Riad HN. Impact of stents on urological complications and health care expenditure in renal transplant recipients: results of a prospective, randomized clinical trial. JUrol. 2007;177(6):2260-4.

5. Wilson $\mathrm{CH}$, Bhatti AA, Rix DA, Manas DM. Routine intraoperative stenting for renal transplant recipients. Transplantation. 2005;80(7):877-82.

6. DuBay DA, Lynch R, Cohn J, Ads Y, Punch JD, Pelletier SJ, et al. Is routine ureteral stenting cost-effective in renal transplantation? J Urol. 2007;178(6):2509-13.

7. Osman Y, Ali-El-Dein B, Shokeir AA, Kamal M, El-Din AB. Routine insertion of ureteral stent in live-donor renal transplantation: is it worthwhile? Urology. 2005;65(5):867-71.

8. Ranganathan M, Akbar M, Ilham MA, Chavez R, Kumar N, Asderakis A. Infective complications associated with ureteral stents in renal transplant recipients. Transplant Proc. 2009;41(1):162-4.

9. Kumar A, Verma BS, Srivastava A, Bhandari M, Gupta A, Sharma R. Evaluation of the urological complications of living related renal transplantation at a single center during the last 10 years: impact of the Double-J* stent. J Urol. 2000;164(3 Pt 1):657-60.

10. Simpson CM, Sterne JA, Walker RG, Francis DM, Robertson AJ, 


\section{Vogel Tet al.}

Jones CL. Stent-related ureteric obstruction in paediatric renal transplantation. Pediatr Nephrol. 2006;21(1):79-85.

11. Garner JS, Jarvis WR, Emori TG, Horan TC, Hughes JM. CDC definitions for nosocomial infections, 1988. Am J Infect Control. 1988;16(3):128-40.

12. Bassiri A, Simforoosh N, Gholamrezaie HR. Ureteral complications in 1100 consecutive renal transplants. Transplantat Proc. 2000;32(3):578-9.

13. Neri F, Tsivian M, Coccolini F, Bertelli R, Cavallari G, Nardo B, et al. Urological complications after kidney transplantation: experience of more than 1,000 transplantations. Transplant Proc 2009;41(4):1224-6.

14. Nie ZL, Zhang KQ, Li QS, Jin FS, Zhu FQ, Huo WQ. Urological complications in 1,223 kidney transplantations. Urol Int. 2009;83(3):337-41.

15. Dominguez J, Clase CM, Mahalati K, MacDonald AS, McAlister VC, Belitsky P, et al. Is routine ureteric stenting needed in kidney transplantation? A randomized trial. Transplantation. 2000;70(4):597-601.

16. Berger PM, Diamond JR. Ureteral obstruction as a complication of renal transplantation: a review. J Nephrol.1998;11(1):20-3.

17. Lojanapiwat B, Mital D, Fallon L, Koolpe H, Raja R, Badosa F, et al Management of ureteral stenosis after renal transplantation. J Am Coll Surg. 1994;179(1):21-4.

18. Rigg KM, Proud G, Taylor RM. Urological complications following renal transplantation. A study of 1016 consecutive transplants from a single centre. Transpl Int. 1994;7(2):120-6.

19. Shoskes DA, Hanbury D, Cranston D, Morris PJ. Urological complications in 1,000 consecutive renal transplant recipients.JUrol. 1995;153(1):18-21.

20. Minnee RC, Bemelman FJ, Laguna Pes PP, ten Berge IJ, Legemate DA, Idu MM. Effectiveness of a 5-day external stenting protocol on urological complications after renal transplantation. World J Surg. 2009;33(12):2722-6. 